\title{
ANALYSIS OF MEIOTIC SEGREGATION PATTERNS AND INTERCHROMOSOMAL EFFECTS IN SPERM FROM 13 ROBERTSONIAN TRANSLOCATIONS
}

\author{
Wang $\mathrm{B}^{1, *}$, Nie $\mathrm{B}^{1, *}$, Tang $\mathrm{D}^{2, *}$, Li $\mathrm{R}^{3, *}$, Liu $\mathrm{X}^{1}$, Song $\mathrm{J}^{1}$, Wang $\mathrm{W}^{1}$, Liu $\mathrm{Z}^{1, * *}$ \\ *These authors contributed equally to this study.
}

\begin{abstract}
**Corresponding Author: Dr. Zhi Liu, Department of Clinical Laboratory, Hubei Maternal and Child Health Hospital, 745 WuLuo Road, Wuhan, Hubei, People's Republic of China. Tel: +86-27-8716-9190. Fax: +86-27-8716-9097. E-mail: wangbo1005@163.com.
\end{abstract}

\begin{abstract}
The frequency of the Robertonian (ROB) translocation in newborn babies is approximately one in 1000. Robertsonian translocation is an unusual type of chromosome rearrangement caused by two particular chromosomes joining together. The aim of the study was to analyze the segregation of the ROB translocations in 13 male carriers, and to verify a possible inter-chromosomal effect (ICE) of the ROB translocation on chromosomes 18, X, and Y. Thirteen male patients were included in the study. Multicolor fluorescent in situ hybridization (FISH) was used to analyze chromosomes 13, 14, 15, 21, 22, 18, X and Y in sperm. Among the heterozygous ROB translocation carriers, the frequency of normal/balanced spermatozoa resulting from alternate segregation varied between 70.4 and $85.2 \%$. The frequency of unbalanced spermatozoa resulting from adjacent segregation varied between 14.8 and $29.6 \%$. Increased frequencies of aneuploidy for a sex chromosome were found in 10 ROB translocation carriers (P2-P8, P10-P12). Increased frequencies of aneuploidy for chromosome 18 were found in 10 ROB translocation carriers (P3-P9, P11-P13). In addition, increased frequencies of diploid were found in 11 ROB translocation carriers (P2-P9, P11-P13). Among the homozygous ROB translocation carriers, the rate of balanced spermatozoa was $99.7 \%$ and the frequency of unbalanced spermatozoa was $0.3 \%$. However, the frequencies of aneuploidy for a sex chromosome and chromosome 18 were normal. Despite the high number of normal/balanced
\end{abstract}

\footnotetext{
${ }^{1}$ Department of Clinical Laboratory, Hubei Maternal and Child Health Hospital, Wuhan, Hubei, People's Republic of China

${ }^{2}$ Department of Clinical Laboratory, Renmin Hospital Wuhan University, Wuhan, Hubei, People's Republic of China

${ }^{3}$ Internal Medicine-Cardiovascular Department, Xiaogan Hospital affiliated to Wuhan University of Science and Technology, Xiaogan, Hubei, People's Republic of China
}

frequencies, there remained many unbalanced spermatozoa resulting from alternate segregation. The ROB translocation carriers may be at an increased risk for ICE. Robertsonian translocation homozygosity could be seen as a potential speciation in humans with 44 chromosomes.

Keywords: Evolution; Interchromosomal effects; Meiotic segregation; Robertsonian (ROB) translocation homozygosity; Sperm fluorescence in situ hybridization (FISH).

\section{INTRODUCTION}

The frequency of the Robertsonian (ROB) translocation in newborn babies is approximately one in 1000 [1]. The ROB translocation is an unusual type of chromosome rearrangement caused by two particular chromosomes joining together. In humans, it occurs in the five acrocentric chromosomes, such as chromosomes 13, 14 and 15 (group D), and 21 and 22 (group G). During a ROB translocation, the participating chromosomes break at their centromeres and the long arms fuse to form a single chromosome with a single centromere. The short arms also join to form a reciprocal products that typically contain nonessential genes and are usually lost within a few cell divisions. The most common ROB translocation in humans is between chromosomes 13 and 14, constituting $75.0 \%$ of all ROB translocations [1]. Since ROB translocation carriers have a balanced chromosomal complement, they may never be aware of their unusual chromosome rearrangement. Therefore, ROB translocation can be passed down in the family for many generations without detection.

In ROB translocations, at the end of meiosis I, segregation of the translocated and non translocated chromosomes from the two different implicated chromosome pairs, lead to the formation of either balanced (alternate segregation mode) or unbalanced (adjacent 1, adjacent 2, and 3:0 segregation modes) gametes that can segre- 
gate in different ways at anaphase [2,3]. Only alternate segregation produces normal/balanced karyotypes. The other segregation modes (adjacent 1, adjacent 2, 3:0) produce unbalanced gametes with disomies and nullisomies of chromosomes involved in ROB translocations. It is well-known that meiotic tetravalent configuration tends to segregate in an alternate way [4], resulting in the production of normal/balanced spermatozoa. However, a certain percentage of unbalanced gametes deriving from adjacent segregation are also produced, and could be responsible for the miscarried or the severely affected aneuploidy offspring frequently born to these carriers.

Analysis of the chromosomal constitution in sperm of ROB translocation carriers is of great interest to assess the risk of unbalanced offspring for genetic counseling. Sperm fluorescence in situ hybridization (FISH) using chromosome specific probes has proven to be a useful technique to determine the meiotic segregation, especially in translocation carriers [5-9]. During the last decade, meiotic segregation in spermatozoa has been repeatedly studied in male carriers of ROB translocations. Despite the large variations, the rate of unbalanced gametes is generally not conclusive enough for genetic counseling. Although most studies showed strong prevalence of alternate segregation, which lead to balanced spermatozoa, one recent study showed a high percentage of unbalanced spermatozoa in two ROB translocation carriers [10].

More recently, inter-chromosomal effects (ICE) have been reported in several chromosome pairs in ROB translocations. Inter-chromosomal effects refer to a disturbance of meiosis, where rearranged chromosomes disrupt dis-junction and distribution of chromosome pairs not involved in the rearrangement. The concept of ICE was first postulated by Lejeune [11], who noticed an excess of carriers of balanced reciprocal translocations among the fathers of children with Down syndrome. Since then, contradictory data have been reported in ROB translocation carriers. Several studies have found such an ICE in male carriers of ROB translocations $[12,13]$, but others did not $[14,15]$. The aims of this study were to analyze the segregation pattern of ROB translocation in 13 male carriers, and evaluate the occurrence of ICE on chromosomes $18, \mathrm{X}$ and $\mathrm{Y}$ in these carriers.

\section{MATERIALS AND METHODS}

Patients. Thirteen male carriers of ROB translocations (designated P1-13) consulting for infertility or repeated abortions were included in this study. Twelve were heterozygous ROB translocation and one was a homozygous ROB translocation. Except P1 and P2 (P2 was P1's father), all the patients were unrelated. Three males with normal 46,XY karyotype and normal sperm characteristics were recruited as controls. The local ethics committee approved this protocol and all patients and controls had previously given informed consent for the study.

Sperm Preparation, Fluorescent in Situ Hybridization and Scoring. All semen samples were first analyzed to evaluate volume, concentration and motility according to the World Health Organization (WHO) criteria [16]. After semen analysis, sperm with progressive motility was isolated and washed twice in phosphate buffered saline $(\mathrm{pH}$ 7.4) by centrifugation at $1500 \mathrm{rpm}$ for $11 \mathrm{~min}$. Final pellets were fixed with $5 \mathrm{~mL}$ of acetic acid/methanol mixture (1:3) for at least $30 \mathrm{~min}$. at $4{ }^{\circ} \mathrm{C}$. Aliquots $(40-50 \mu \mathrm{L})$ of the resulting suspension of nuclei were smeared on cold pre-cleaned slides. Nuclei decondensation was performed in $1 \mathrm{~N} \mathrm{NaOH}$ for $2 \mathrm{~min}$. After dehydration in ethanol series $(70.0,90.0,100.0 \%)$, denaturation was performed in $0.25 \%$ formamide in $2 \times$ saline sodium citrate (SSC) followed by overnight hybridization with a combination of commercially available probes.

To assess the segregation behavior of each rearrangement, a dual-color FISH using locus-specific probes and/ or telomere probes and/or chromosome enumeration probes, was carried out on spermatozoa of the translocation carriers. Below we list the probes that we used to identify the segregation products for each karyotype. For a der(13;14) carrier, LSI13 (13q14, Spectrum Green) and Tel14 (14q32.33, Spectrum Red) were used. For a $\operatorname{der}(14 ; 15)$ carrier, Tel14 (14q32.33, Spectrum Red) and Tel15 (15q26.3, Spectrum Green) were used. For a der(14;21) carrier, Tel14 (14q32.33, Spectrum Red) and LSI21 (21q22.13-q22.2, Spectrum Green) were used. For a der(13;22) carrier, LSI13 (13q14, Spectrum Green) and LSI22 (22q12.3, Spectrum Red) were used. For a $\operatorname{der}(21 ; 22)$ carrier, LSI21 (21q22.13-q22.2, Spectrum Green) and LSI22 (22q12.3, Spectrum Red) were used. Regarding ICE evaluation, triple-color FISH was performed using the probe mixture including commercial satellite (DNA) probes for chromosomes 18, X and Y (CEP 18, Spectrum Blue/CEP X, Spectrum Green/CEP Y, Spectrum Red) from Vysis (Abbott Park, IL, USA).

Post-hybridization washes included $2 \mathrm{~min}$. in $0.4 \times$ $\mathrm{SSC} / 0.3 \% \mathrm{NP}-40(\mathrm{pH} 7)$ at $72{ }^{\circ} \mathrm{C}$, followed by $1 \mathrm{~min}$. in $2 \times \mathrm{SSC} / 0.1 \% \mathrm{NP}-40(\mathrm{pH} 7)$ at room temperature. Slides were covered with DAPI II (Vysis). Only intact spermatozoa bearing a similar degree of decondensation and clear hybridization signals were scored. Disrupted or overlapping spermatozoa were excluded from analysis. Only slides with hybridization efficiency of $99.0 \%$ and more were analyzed. One thousand sperm nuclei per patient were analyzed. 
Statistical analyses. The $\chi^{2}$ test was used to compare the frequencies of segregation products. A $p$ value of less than 0.05 was regarded as statistically significant.

\section{RESULTS}

The age of our patients ranged from 25 to 48 years with a mean of 38.6 years. The karyotype and sperm examination of patients and controls are given in Table 1 . According to WHO criteria, all our patients, except P1, had very poor semen parameters. The karyotyping analysis identified a previously undescribed balanced human karyotype 44,XY,der(14;15)(q10;q10),der(14;15)(q10;q10) (Figure 1). Interestingly, in P1, we saw a sperm (arrow) with four fluorescence signals (two red and two green). This means the sperm has two derivative chromosomes of $\operatorname{der}(14 ; 15)$, but we could not tell if the sperm was a disomy of derivative chromosome or diploid (Figure 2). In future studies, we will employ triple-color FISH (for example, the probe mixture will consist of chromosomes 14, 15 and 18)

Table 1. Cytogenetic and spermiologic results of patients and controls.

\begin{tabular}{|c|c|c|c|c|}
\hline Patient & Karyotype & $\begin{array}{c}\text { Age } \\
\text { (years) }\end{array}$ & $\begin{array}{c}\text { Sperm Concentration } \\
\left(\mathbf{1 0}^{6} / \mathbf{L}\right)\end{array}$ & $\begin{array}{l}\text { Motility }^{\mathbf{2}} \\
(\mathbf{a}+\mathbf{b}) \%\end{array}$ \\
\hline P1 & 44,XY,der(14;15)(q10;q10),der(14;15)(q10;q10) & 25 & 56.0 & 53.0 \\
\hline $\mathrm{P} 2$ & $45, X Y, \operatorname{der}(14 ; 15)(q 10 ; q 10)$ & 48 & 13.0 & 22.0 \\
\hline P3 & 45,XY,der(13;14)(q10;q10) & 29 & 19.0 & 25.0 \\
\hline P4 & 45,XY,der(13;14)(q10;q10) & 31 & 22.0 & 17.0 \\
\hline P5 & 45,XY,der(13;14)(q10;q10) & 27 & 38.0 & 21.0 \\
\hline P6 & 45,XY,der(13;14)(q10;q10) & 35 & 57.0 & 59.0 \\
\hline P7 & 45,XY,der(13;14)(q10;q10) & 33 & 89.0 & 38.0 \\
\hline P8 & $45, X Y, \operatorname{der}(13 ; 14)(q 10 ; q 10)$ & 30 & 35.0 & 29.0 \\
\hline $\mathrm{P} 9$ & 45,XY,der(14;21)(q10;q10) & 36 & 40.0 & 35.0 \\
\hline $\mathrm{P} 10$ & 45,XY,der(14;21)(q10;q10) & 32 & 37.0 & 36.0 \\
\hline P11 & 45,XY,der(14;21)(q10;q10) & 26 & 42.0 & 45.0 \\
\hline P12 & $45, X Y, \operatorname{der}(13 ; 22)(q 10 ; q 10)$ & 26 & 28.0 & 39.0 \\
\hline $\mathrm{P} 13$ & 45,XY,der(21;22)(q10;q10) & 29 & 31.0 & 47.0 \\
\hline $\mathrm{C} 1$ & $46, X Y$ & 35 & 77.0 & 44.0 \\
\hline $\mathrm{C} 2$ & $46, X Y$ & 27 & 62.0 & 47.0 \\
\hline $\mathrm{C} 3$ & $46, X Y$ & 33 & 43.0 & 39.0 \\
\hline
\end{tabular}

${ }^{a}$ Sperm motility was graded from a to $\mathrm{d}$, according to the WHO manual criteria, as follows. Grade a (fast progressive) sperms are those that swim forward fast in a straight line, like guided missiles. Grade b (slow progressive) sperms swim forward, but either in a curved or crooked line, or slowly (slow linear or non linear motility). Grade c (non progressive) sperms move their tails, but do not move forward (local motility only). Grade d (immotile) sperms do not move at all. The $(\mathrm{a}+\mathrm{b}) \%$ represent the percentage of sperm that can move forward normally.

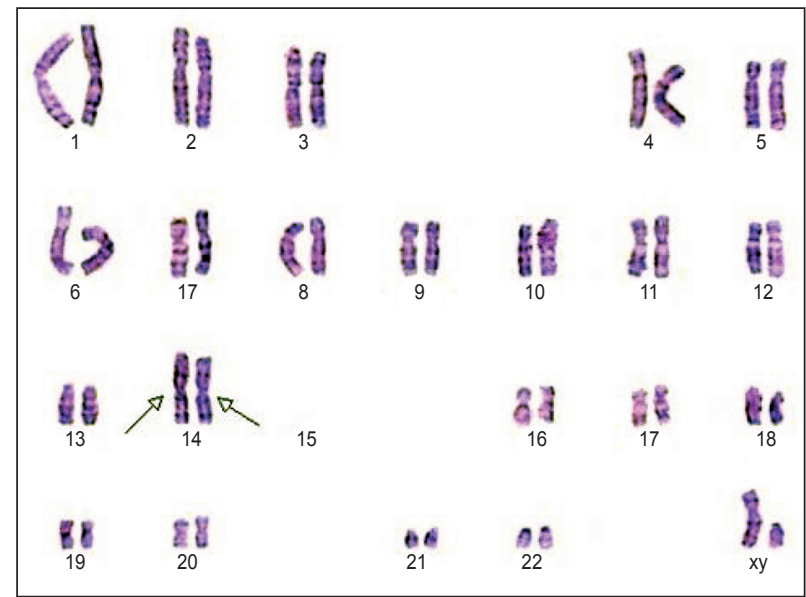

Figure 1. Karyotype of P1: 44,XY,der(14;15)(q10; $\mathrm{q} 10), \operatorname{der}(14 ; 15)(\mathrm{q} 10 ; \mathrm{q} 10)$, carrying a disomy for the ROB translocation chromosome (highlighted by arrows).

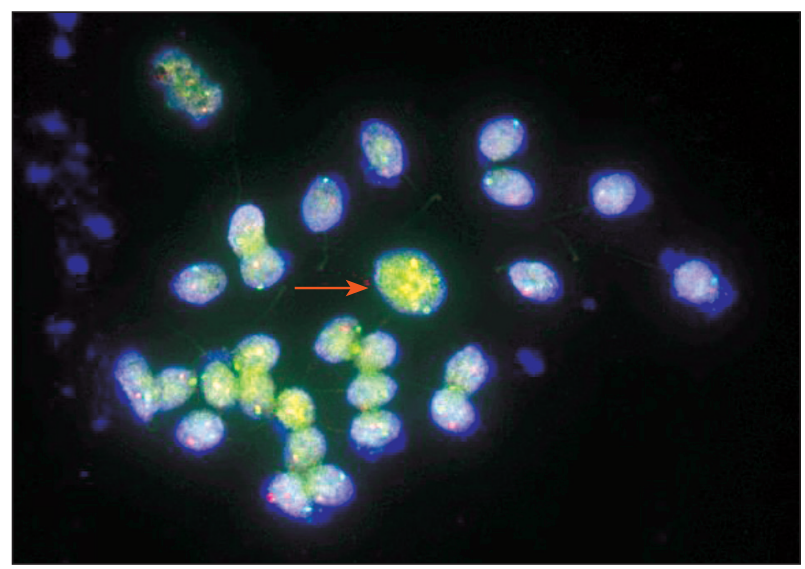

Figure 2. Sperm cells after hybridization with TelVysion 14q32.33 (Spectrum Red) and TelVysion 15q26.3 (Spectrum Green). The sperm highlighted with a red arrow may be a diploid sperm. 
Table 2. The number of spermatozoa scored, the alternate mode of segregation, incidence of sperm nullisomy, disomy and 3:0/ diploid for the chromosomes involved in the Robertsonian translocation in 13 Robertsonian translocation carriers and three controls.

\begin{tabular}{|l|c|c|c|c|c|c|c|c|}
\hline & $\begin{array}{c}\text { Normal/ } \\
\text { Balanced }\end{array}$ & Nullisomy A & Disomy A & Nullisomy B & Disomy B & $\begin{array}{c}\mathbf{3 : 0} \text { or } \\
\text { Diploid }\end{array}$ & Unbalanced & Total \\
\hline P1 & 997 & 2 & 0 & 0 & 0 & 1 & 3 & 1000 \\
\hline P2 & 799 & 41 & 49 & 55 & 45 & 11 & 201 & 1000 \\
\hline P3 & 852 & 24 & 57 & 34 & 26 & 7 & 148 & 1000 \\
\hline P4 & 809 & 68 & 30 & 55 & 29 & 9 & 191 & 1000 \\
\hline P5 & 843 & 40 & 39 & 27 & 41 & 10 & 157 & 1000 \\
\hline P6 & 757 & 55 & 37 & 94 & 42 & 15 & 243 & 1000 \\
\hline P7 & 837 & 29 & 66 & 34 & 21 & 13 & 163 & 1000 \\
\hline P8 & 782 & 75 & 61 & 49 & 22 & 11 & 218 & 1000 \\
\hline D/D mean & 811 & 47.0 & 48.0 & 50.0 & 32.0 & 11.0 & 189.0 & \\
\hline P9 & 775 & 41 & 50 & 70 & 45 & 19 & 225 & 1000 \\
\hline P10 & 721 & 66 & 37 & 110 & 45 & 21 & 279 & 1000 \\
\hline P11 & 704 & 47 & 56 & 73 & 95 & 25 & 296 & 1000 \\
\hline P12 & 719 & 69 & 75 & 62 & 58 & 17 & 281 & 1000 \\
\hline D/G mean & 730 & 56.0 & 55.0 & 79.0 & 61.0 & 21.0 & 270.0 & \\
\hline P13 & 768 & 47 & 51 & 42 & 80 & 12 & 232 & 1000 \\
\hline C1 & 995 & 0 & 2 & 1 & 1 & 1 & 5 & 1000 \\
\hline C2 & 997 & 1 & 0 & 2 & 0 & 0 & 3 & 1000 \\
\hline C3 & 995 & 0 & 2 & 0 & 1 & 2 & 5 & 1000 \\
\hline Control mean & 996 & 0.3 & 1.3 & 1.0 & 0.7 & 1.0 & 4.3 & \\
\hline
\end{tabular}

A: first chromosome involved in the ROB translocation; B: second chromosome involved in the ROB translocation; D/D mean: the mean of P2-P8 (ROB translocation between D/D groups); D/G mean: the mean of P9-P12 (ROB translocation between D/G groups);

Control mean: the mean of the three controls (C1-C3).

Table 3. Incidence of sperm nullisomy, disomy and diploid for chromosomes $18, \mathrm{X}$ and $\mathrm{Y}$ in 13 Robertsonian translocation carriers and three controls.

\begin{tabular}{|c|c|c|c|c|c|c|c|c|}
\hline & $\begin{array}{l}\text { Normal/ } \\
\text { Balanced }\end{array}$ & $\begin{array}{c}\text { Nullisomy } \\
18\end{array}$ & $\begin{array}{c}\text { Disomy } \\
18\end{array}$ & $\begin{array}{l}\text { Nullisomy Sex } \\
\text { Chromosome }\end{array}$ & $\begin{array}{c}\text { Disomy Sex } \\
\text { Chromosome }\end{array}$ & $\begin{array}{l}\text { 3:0 or } \\
\text { Diploid }\end{array}$ & Unbalanced & Total \\
\hline P1 & 994 & 0 & 1 & 2 & 3 & 0 & 6 & 1000 \\
\hline $\mathrm{P} 2$ & 949 & 3 & 2 & 15 & 10 & 21 & 41 & 1000 \\
\hline P3 & 899 & 6 & 5 & 22 & 21 & 47 & 101 & 1000 \\
\hline P4 & 952 & 4 & 5 & 17 & 12 & 10 & 48 & 1000 \\
\hline P5 & 913 & 11 & 9 & 13 & 15 & 39 & 87 & 1000 \\
\hline P6 & 947 & 7 & 2 & 15 & 17 & 12 & 53 & 1000 \\
\hline P7 & 955 & 9 & 12 & 10 & 5 & 9 & 45 & 1000 \\
\hline P8 & 901 & 21 & 13 & 24 & 19 & 22 & 99 & 1000 \\
\hline $\mathrm{D} / \mathrm{D}$ mean & 931 & 8.7 & 6.9 & 16.6 & 14.1 & 22.9 & 67.7 & \\
\hline P9 & 976 & 3 & 4 & 4 & 2 & 11 & 24 & 1000 \\
\hline P10 & 985 & 2 & 1 & 3 & 6 & 3 & 15 & 1000 \\
\hline P11 & 963 & 6 & 4 & 9 & 11 & 7 & 37 & 1000 \\
\hline $\mathrm{P} 12$ & 944 & 7 & 5 & 8 & 11 & 25 & 56 & 1000 \\
\hline $\mathrm{D} / \mathrm{G}$ mean & 967 & 4.5 & 3.5 & 6.0 & 7.5 & 11.5 & 33.0 & \\
\hline P13 & 977 & 7 & 6 & 1 & 4 & 5 & 23 & 1000 \\
\hline $\mathrm{C} 1$ & 995 & 0 & 2 & 1 & 1 & 1 & 5 & 1000 \\
\hline $\mathrm{C} 2$ & 997 & 1 & 0 & 2 & 0 & 0 & 3 & 1000 \\
\hline $\mathrm{C} 3$ & 991 & 0 & 2 & 3 & 1 & 3 & 9 & 1000 \\
\hline Control mean & 994 & 0.3 & 1.3 & 2.0 & 0.7 & 1.3 & 5.7 & \\
\hline
\end{tabular}


for the detection of normal/balanced or unbalanced sperm to distinguish between disomy and diploid.

The results of the segregation analysis are given in Table 2 and Table 3. Among the 13 ROB translocation carriers, one was homozygous (P1). The remainder were heterozygous (P2-P13). The frequency of balanced spermatozoa was $99.7 \%$ in P1, while the frequency of unbalanced spermatozoa was $0.3 \%$, which was similar to the controls [17-21] (Table 2).

In P2-P13, the frequency of normal/balanced spermatozoa resulting from alternate segregation varied between 70.4 and $85.2 \%$. The frequency of unbalanced spermatozoa resulting from adjacent segregation varied between 14.8 and $29.6 \%$. The frequency of unbalanced spermatozoa was significantly higher than P1 and the controls $(p<0.05)$.

To evaluate the occurrence of ICE, the nullisomy, disomy and diploid frequencies for chromosomes 18, X and $\mathrm{Y}$ are summarized in Table 3. Increased frequencies of aneuploidy for a sex chromosome were observed in 10 ROB translocation carriers (P2-P8, P10-P12), Increased frequencies of aneuploidy for chromosome 18 were observed in 10 ROB translocation carriers (P3-P9, P11-P13). In addition, increased frequencies of diploid were found in 11 ROB translocation carriers (P2-P9, P11-P13). Increased frequencies of unbalanced sperms were found in heterozygous carriers (P2-P13). In contrast, no ICE was detected in P1.

\section{DISCUSSION}

Sperm chromatin is a highly organized, compact structure consisting of DNA and heterogeneous nucleoproteins, which are essential for the accurate transmission of genetic information to offspring [22]. Sperm FISH is most commonly used to determine the proportion of aneuploidy present in sex chromosomes and autosomes of infertile men. Furthermore, it can also quantify the probability of transmitting aneuploidies and complex chromosomal rearrangements, such as translocations and inversions [22]. As a result, sperm FISH analysis is increasingly included in infertility diagnostic protocols, providing useful information for genetic consulting. In our study, we performed sperm FISH analyses on 13 male ROB translocation carriers.

In our study, we had one homozygous carrier (P1) and 12 heterozygous carriers (P2-P13). In P1, the frequency of unbalanced spermatozoa was only $0.3 \%$ that was similar to controls and is consistent with previous publications [5-7]. In contrast, in P2-P13, the frequency of normal/ balanced spermatozoa resulting from alternate segregation varied between 70.4 and $85.2 \%$ (Table 2). This is consistent with previous studies showing that alternate segregation is predominant in sperm of Robertsonian translocation carriers $[11,20,21]$. The high prevalence of the alternate segregation is presumably due to cis-configuration of the trivalent during meiosis, which favors an alternate segregation in all ROB translocations [21-24]. However, the frequency of unbalanced spermatozoa resulting from adjacent segregation varied between 14.8 and 29.6\% (Table 2 ), which is significantly higher than controls. Robertsonian translocations consist of a fusion of two acrocentric chromosomes at the centromere level to form a derivative chromosome (group D: 13, 14 and 15; group G: 21 and 22). Robertsonian translocation can be separated into three subtypes: D/D, D/G and G/G translocation. Patients P2P8 were D/D ROB translocation carriers; P13 was a G/G ROB translocation carrier, and P9-P12 were D/G carriers. The frequency of unbalanced spermatozoa was highest in the $\mathrm{D} / \mathrm{G}$ carriers and lowest in the $\mathrm{D} / \mathrm{D}$ carriers and the differences between $\mathrm{D} / \mathrm{D}$ and $\mathrm{D} / \mathrm{G}, \mathrm{D} / \mathrm{D}$ and $\mathrm{G} / \mathrm{G}$ were significant $(p<0.05)$. In contrast, the difference between $\mathrm{G} / \mathrm{G}$ and $\mathrm{D} / \mathrm{G}$ was not significant $(p>0.05)$. This is consistent with previous publications $[6,17]$. We speculated that in D/D ROB translocation, the derivative chromosome is a metacentric chromosome, so it is prone to alternate segregation rather than adjacent segregation at the end of meiosis I. In the G/G ROB translocation, the derivative chromosome is a metacentric chromosome, but the derivative chromosome is shorter than the derivative chromosome of $\mathrm{D} / \mathrm{D}$ ROB translocation, so the rate of adjacent segregation is higher than $\mathrm{D} / \mathrm{D}$ ROB translocation. In the $\mathrm{D} / \mathrm{G}$ ROB translocation, the derivative chromosome is a submetacentric chromosome, it is more chaotic than $\mathrm{D} / \mathrm{D}$ and $\mathrm{G} / \mathrm{G}$ ROB translocations at the end of meiosis I, so the rate of unbalanced spermatozoa is significantly higher than $\mathrm{D} / \mathrm{D}$ and $\mathrm{G} / \mathrm{G}$ ROB translocations.

The homozygous ROB translocation carrier (P1) is healthy and has a balanced chromosomal complement. Assessment of a semen sample from P1 showed normal sperm number and morphology. Given his karyotype of 44,XY,der(14;15)(q10;q10),der(14;15)(q10;q10), we hypothesized that the person's sperm karyotype to be consistently 22,X,der(14;15)and 22,Y,der(14;15), then, our hypothesis was proven by this research. Most of the sperm from P1 are balanced haploid (not normal haploid) (Table 2, Table 3). Homozygous ROB translocations in man have been described before. A fetus with two t $(14 ; 21)$ chromosomes was found by Dallapiccola et al. [25]. The related parents were heterozygous for the same translocation. Martinez et al. [26] described three adult siblings homozygous for $\mathrm{t}(13 ; 14)$. Their parents were first cousins and both 
were heterozygous carriers. Rajangam et al. [27] found a unique DS karyotype 45,XY,der(14;21)pat,der(14;21)mat, +21 mat. While translocation heterozygosity is associated with meiotic disturbances that cause infertility and subfecundity, translocation homozygosity should not, at least in theory, have any effect on meiosis [28]. In our study, we have one patient with homozygous ROB translocation.

Inter-chromosomal effect remains controversial in the literature [29]. Some publications have indicated its relevance [12,13], while others showed no evidence of ICE in ROB translocation carriers $[9,18]$. Inter-chromosomal effect could be explained by the formation of heterosynapses between chromosomes involved in the translocation and the sex vesicle, which could also involve other chromosomes [11,29]. In our study, we observed higher frequency of aneuploidy for the sex chromosome in 10 ROB translocation carriers (P2-P8, P10-P12) and higher frequencies of aneuploidy for chromosome 18 in 10 ROB translocation carriers (P3-P9, P11-P13). In addition, increased rates of diploid were found in 11 ROB translocation carriers (P2P9, P11-P13). Increased rates of unbalanced sperms were found in all ROB translocation heterozygote (P2-P13). The incidence of spermatozoa with nullisomy, disomy and diploid for the sex chromosomes of ROB translocation heterozygotes (P2-P13) was significantly higher compared to that of ROB translocation homozygosity (P1) $(p<0.05)$. Robertsonian translocation rearrangements are common chromosomal changes that can lead to rapid and efficient reproductive isolation between karyotypically similar populations, especially when many ROB metacentric chromosomes display monobrachial homologies [30]. In the case of Muntjac deer, also called Cervidae, which are of great interest to evolutionary biologists and cytogeneticists because of the considerable diversity of their karyotypes, despite their morphological similarity [28].

To conclude, our study confirmed that alternate segregation is dominant in ROB translocation carriers. However, despite the high number of normal/balanced spermatozoa, there are still a lot unbalanced spermatozoa resulting from adjacent mode of segregation. Our study provides further evidence of ICE on chromosome X, Y and 18 in ROB translocation carriers. Therefore, preimplantation genetic diagnosis (PGD) is recommended for ROB translocation carriers to minimize the risk of having an affected child or the distress of pregnancy termination, and to reduce the risk of miscarriage due to abnormal segregation of the translocation [31-34]. Since the proband is phenotypically normal with normal fertility, we considered the chromosomal rearrangement of the person to be a balanced polymorphism [35]. The aberration can provide material for evolution. The establishment of a new human subspecies with a diploid complement of 44 chromosomes could occur if a small population with the karyotype of the proband undergoes long-term reproductive isolation [36].

\section{ACKNOWLEDGMENTS}

This study was supported by National Science Foundation of China (Grant No. 81102141) and Youth Science Foundation of Hubei Province (Grant No. WJ2015Q015)

Declaration of Interest. The authors report no conflicts of interest. The authors alone are responsible for the content and writing of this article.

\section{REFERENCES}

1. Therman E, Susman B, Denniston C. The nonrandom participation of human acrocentric chromosomes in Robertsonian translocations. Ann Hum Genet. 1989; 53(1): 49-65.

2. Luciani JM, Guichaoua MR, Mattei A, Morazzani MR. Pachytene analysis of a man with a $13 \mathrm{q} ; 14 \mathrm{q}$ translocation and infertility. Cytogenet Cel Genet. 1984; 38(1): 14-22.

3. Cassuto NG, Le Foll N, Chantot-Bastaraud S, Balet $\mathrm{R}$, Bouret D, Rouen A, et al. Sperm fluorescence in situ hybridization study in nine men carrying a Robertsonian or a reciprocal translocation: Relationship between segregation modes and high-magnification sperm morphology examination. Fertil Steril. 2011; 96(4): 826-832.

4. Sybenga J. Chromosome structural variants. In: Sybenga J, Ed. General Cytogenetics. Amsterdam, The Netherlands: North-Holland Publishing Company 1975: 165-212.

5. Roux C, Tripogney C, Morel F, Joanne C, Fellmann $\mathrm{F}$, Clavequin MC, et al. Segregation of chromosomes in sperm of Robertsonian translocation carriers. Cytogenet Genome Res. 2005; 111(3-4): 291-296.

6. Rives N, Ravel C, Duchesne V, Siffroi JP, MoussetSimeon N, Mace B. Molecular cytogenetics analysis with whole chromosome paint probes of sperm nuclei from a $(13 ; 15)$ Robertsonian translocation carrier. J Hum Genet. 2005; 50(7): 360-364.

7. Anahory T, Hamamah S, Andreo B, Hedon B, Claustres M, Sarda P, et al. Sperm segregation analysis of a $(13 ; 22)$ Robertsonian translocation carrier by FISH: 
A comparison of locus-specific probe and whole chromosome painting. Hum Reprod. 2005; 20(7): 1850-1854.

8. Moradkhani K, Puechberty J, Bhatt S, Vago P, Janny $\mathrm{L}$, Lefort $\mathrm{G}$, et al. Meiotic segregation of rare Rob-ertsonian translocations: Sperm analysis of three $\mathrm{t}(14 \mathrm{q}$; 22q) cases. Hum Reprod. 2006; 21(5): 1166-1171.

9. Anton E, Vidal F, Blanco J. Role of sperm FISH studies in the genetic reproductive advice of structural reorganization carriers. Hum Reprod. 2007; 22(8): 2088-2092.

10. Liu Y, Zhu H. Detection of sperm chromosomes in Robertsonian translocation carriers by dual-color fluorescence in situ hybridization. Zhonghua Nan Ke Xue. 2004; 10(2): 90-93.

11. Lejeune J. Autosomal disorders. Pediatrics. 1963; 32(3): 326-337.

12. Douet-Guilbert N, Bris MJ, Amice V, Marchetti C, Delobel B, Amice J, et al. Interchromosomal effect in sperm of males with translocations: Report of 6 cases and review of the literature. Int J Androl. 2005; 28(6): 372-379.

13. Ogur G, Van Assche E, Vegetti W, Verheyen G, Tournaye $\mathrm{H}$, Bonduelle $\mathrm{M}$, et al. Chromosomal segregation in spermatozoa of 14 Robertsonian translocation carriers. Mol Hum Reprod. 2006; 12(3): 209-215.

14. Estop AM, Cieply K, Munne S, Surti U, Wakim A, Feingold E. Is there an interchromosomal effect in reciprocal translocation carriers? Sperm FISH studies. Hum Genet. 2000; 106(5): 517-524.

15. Acar H, Yildirim MS, Cora T, Ceylaner S. Evaluation of segregation patterns of 21;21 Robertsonian translocation along with sex chromosomes and interchromosomal effects in sperm nuclei of carrier by FISH technique. Mol Reprod Dev. 2002; 63(2): 232-236.

16. World Health Organisation. WHO Laboratory Manual for the Examination and Processing of Human Semen, 5th ed. Geneva, Switzerland, 2010.

17. Machev N, Gosset P, Warter S, Treger M, Schillinger $\mathrm{M}$, Viville S. Fluorescence in situ hybridization sperm analysis of six translocation carriers provides evidence of an interchromosomal effect. Fertil Steril. 2005; 84(2): 365-373.

18. Mahjoub M, Mehdi M, Brahem S, Elghezal H, Ibala $\mathrm{S}$, Saad A. Chromosomal segregation in spermatozoa of five Robertsonian translocation carriers $\mathrm{t}(13 ; 14)$. J Assist Reprod Genet. 2011; 28(7): 607-613.
19. Rouen A, Pyram K, Pollet-Villard X, Hyon C, Dorna $\mathrm{M}$, Marques S, et al. Simultaneous cell by cell study of both DNA fragmentation and chromosomal segregation in spermatozoa from chromosomal rearrangement carriers. J Assist Reprod Genet. 2013;30(3):383390. doi: $10.1007 /$ s 10815-012-9915-7.

20. Ferfouri F, Selva J, Boitrelle F, Gomes DM, Torre A, AlbertM, et al. The chromosomal risk in sperm from heterozygous Robertsonian translocation carriers is related to the sperm count and the translocation type. Fertil Steril. 2011; 96(6): 1337-1343.

21. Kovac JR, Pastuszak AW, Lamb DJ. The use of genomics, proteomics, and metabolomics in identifying biomarkers of male infertility. Fertil Steril. 2013; 99(4): 998-1007.

22. Luciani JM, Guichaoua MR, Mattei A, Morazzani MR. Pachytene analysis of a man with a $13 \mathrm{q} ; 14 \mathrm{q}$ translocation and infertility. Behavior of the trivalent and nonrandom association with the sex vesicle. Cytogenet Cell Genet. 1984; 38(1): 14-22.

23. Navarro J, Vidal F, Benet J, Templado C, Marinas Egozcue J. XY-trivalent association and synaptic anomalies in a male carrier of a Robertsonian $\mathrm{t}(13 ; 14)$ translocation. Hum Reprod. 1991; 6(3): 376-381.

24. Morel F, Douet-Guilbert N, Le Bris MJ, Herry A, Amice V, Amice J, et al. Meiotic segregation of translocations during male gametogenesis. Int J Androl. 2004; 27(4): 200-212.

25. Dallapiccola B, Ferranti G, Altissimi D, Colloridi F, Paesano R. First-trimester prenatal diagnosis of homozygous $(14 ; 21)$ translocation in a fetus with 44 chromosomes. Prenat Diagn. 1989; 9(8): 555-558.

26. Martinez-Castro P, Ramos MC, Rey JA, Benitez J, Sanchez Cascos A. Homozygosity for a Robertsonian-translocation $(13 q 14 q)$ in three offspring of heterozygous parents. Cytogenet Cell Genet. 1984; 38(4): 310-312.

27. Rajangam S, Michaelis RC, Velagaleti GV, Lincoln $\mathrm{S}$, Hegde $\mathrm{S}$, Lewin $\mathrm{S}$, et al. Down syndrome with biparental inheritance of der(14q21q) and maternally derived trisomy 21: Confirmation by fluorescent in situ hybridization and microsatellite polymorphism analysis. Am J Med Genet Part A. 1997; 70(1): 43-47.

28. Wang W, Lan H. Rapid and parallel chromosomal number reductions in muntjac deer inferred from mitochondrial DNA phylogeny. Mol Biol Evol. 2000; 17(9): 1326-1333.

29. Lindenbaum RH, Hulten M, McDermott A, Seabright $\mathrm{M}$. The prevalence of translocations in parents of chil- 
dren with regular trisomy 21: A possible interchromosomal effect? J Med Genet. 1985; 22(1): 24-28.

30. Britton-Davidian J, Catalan J, da Graça Ramalhinho M, Ganem G, Auffray JC, Capela R, et al. Rapid chromosomal evolution in island mice. Nature. 2000; 403(6766): 158.

31. Bint SM, Makie Ogilvie C, Flinter FA, Khalaf Y, Scriven PN. Meiotic segregation of Robertsonian translocations ascertained in cleavage-stage embryos - Implications for preimplantation genetic diagnosis. Hum Reprod. 2011: 26(6): 1575-1584.

32. Bernicot I, Schneider A, Mace A, Hamamah S, Hedon $\mathrm{B}$, Pellestor F, et al. Analysis using fish of sperm and embryos from two carriers of rare rob $(13 ; 21)$ and rob(15; 22) robertsonian translocation undergoing PGD. Eur J Med Genet. 2012; 55(4?): 245-251.
33. Fischer J, Colls P, Escudero T, Munne S. Preim-plantation genetic diagnosis (PGD) improves pregnancy outcome for translocation carriers with a history of recurrent losses. Fertil Steril. 2010; 94(1): 283-289.

34. Jin H, Ping L, Jie Q, Ying L, Jongjian C. Translocation chromosome karyotypes of the Robertsonian trans-location carriers' embryos. Fertil Steril. 2010; 93(4): 1061-1065.

35. John B. Chromosome change and evolutionary change: A critique. In: Atchley WR, Woodruff DS, Eds. Evolution and Specification: Essays in Honor of M.J.D. White. London, UK: Cambridge University Press. 1981: 23-51.

36. Wang B, Xia Y, Song J, Wang W, Tang Y. Potential speciation in humans involving Robertsonian translocations. Biomed Res. 2013; 24(1): 171-174 